\title{
Expression of angiogenic growth factors VEGF, bFGF and ANG1 in colon cancer after bevacizumab treatment in vitro: A potential self-regulating mechanism
}

\author{
MINJIAN ZHAO, ZHAOSHI YU, ZHIHONG LI, JUN TANG, XU LAI and LIMING LIU \\ Department of General Surgery, Central Hospital of Ezhou, Hubei 436000, P.R. China
}

Received May 16, 2016; Accepted July 25, 2016

DOI: $10.3892 / o r .2016 .5231$

\begin{abstract}
The present study aimed to examine changes in the expression of angiogenic growth factors in vascular endothelial cells isolated from colon cancer after bevacizumab treatment in vitro, and to explore a potential mechanism of their selfregulation as a possible mechanism for antiangiogenic therapy failure in clinics. Vascular endothelial cells were isolated from tumors of colon cancer patients and transfected with recombinant adeno-associated virus type 2-vascular endothelial growth factor (VEGF) or pGIPZ-VEGF RNA interference in order to upregulate or downregulate VEGF expression. Changes in VEGF expression and its correlation with the expression of angiogenesis-related factors, including basic fibroblast growth factor (bFGF) and angiopoietin 1 (ANG1), after treatment with bevacizumab in vitro, were investigated. The results showed that in cells with VEGF overexpression, bFGF and ANG1 were downregulated, whereas in cells in which VEGF was knocked down, upregulation of bFGF and ANG1 was detected. In cells treated with bevacizumab, a significant upregulation of VEGF and downregulation of bFGF and ANG1 were observed. Our data indicate that after bevacizumab treatment, a potential self-regulating mechanism of angiogenic growth factors in colon cancer-derived endothelial cells is activated, which may explain why current antiangiogenic therapy with bevacizumab has limited effects in prolonging the survival of colon cancer patients.
\end{abstract}

\section{Introduction}

Colon cancer is a common abdominal tumor. Despite advances in the understanding of its molecular ethiopathology, diagnosis and treatment, colon cancer still remains one of the leading causes of cancer-related death, particularly when diagnosed in its later stages (1). Angiogenesis, the formation of new

Correspondence to: Professor Liming Liu, Department of General Surgery, Central Hospital of Ezhou, Hubei 436000, P.R. China E-mail: zhf256@163.com

Key words: colon cancer, bevacizumab, VEGF, bFGF, ANG1, selfregulating mechanism vessels, has been postulated as one of the hallmarks of cancer. It is important both in early stages of tumor growth and in its metastatic spread (2). This renders it an attractive target for antitumor therapy. Indeed, the first successful antiangiogenic drug, bevacizumab, a monoclonal antibody against vascular endothelial growth factor (VEGF), has been used in the treatment of advanced colon cancer for more than a decade (3). Despite initially promising results and wider therapeutic indications for its use, including with several other types of cancer, recent studies indicate that bevacizumab therapy has had limited results in increasing the survival of colon cancer patients (4-6).

Angiogenesis is a complex process, in which many growth factors, such as VEGF (7-10), angiopoietin 1 (ANG1), basic fibroblast growth factor (bFGF), among many others, are involved. These factors are in a continuous dynamic balance in order to successfully maintain the homeostasis of an organism. VEGF is a key mediator of angiogenesis $(8,11)$, and bevacizumab inhibits its physiological function by binding to it, and thus preventing its interaction with its receptor and further activation of signaling pathways (11). Why then does colon cancer therapy with bevacizumab fail in the long run? One possible explanation is that the tumor activates collateral signaling pathways in order to overcome angiogenesis inhibition caused by bevacizumab.

Systems biology offers new ways to study neovascularization. It is known that stable system processes have some common features, such as for instance self-regulating mechanisms that govern system formation and stability (12-14). Indeed, growth factors involved in angiogenesis form complex self-regulatory networks maintaining the dynamic stability of the system $(15,16)$. Therefore, we decided to examine whether there is a self-regulating mechanism in colon cancer vessels that abolishes the effects of angiogenesis inhibition with bezvacizumab in the long run. Whether it exists, in the case of VEGF inhibition, the self-regulating mechanism would activate other factors to completely or at least in part compensate for its function, and maintain balanced angiogenesis. In this case, the use of only bevacizumab would not be enough to inhibit the angiogenesis in colon cancer. Exploring these questions would possibly clarify why bevacizumab therapy has had modest results for colon cancer.

Based on this hypothesis in the present study, we decided to examine these potential self-regulating mechanisms in 
bevacizumab-mediated VEGF inhibition in tumor endothelial cells (TECs) isolated from colon tumors in vitro.

\section{Materials and methods}

Tumor endothelial cell culture. Twelve colon cancer patients from the Central Hospital of Ezhou City (Hubei, China), were included in the present study, and cultures of 12 groups of TECs were established from their tumors. We received informed consent from the 12 patients in regards to the present study, and all of the patients signed consent forms. Samples were managed in accordance with the Declaration of Helsinki Guidelines for Research Involving Human Tissue. Before performing any experiments, the established endothelial cells were comfirmed using angiogenesis-antibody array and intracellular signaling array. The protocol was approved by our institutional review board. The donors were colon cancer patients with an average age of 56.3 years. In brief, tumor tissue was harvested and homogenized by two gentle up- and-down strokes in a 15-ml homogenizer (Dounce; Bellco Glass Co., Vineland, NJ, USA). The homogenate was filtered through an $88-\mu \mathrm{m}$ sieve. The remaining retentate was digested in $0.066 \%$ collagenase for $45 \mathrm{~min}$ at $37^{\circ} \mathrm{C}$. The homogenate was centrifuged $(1,000 \mathrm{x} \mathrm{g}$ for $10 \mathrm{~min})$, and the pellet was resuspended in endothelial-basal growth medium-free of human serum (Invitrogen-Gibco, Grand Island, NY, USA), supplemented with $20 \%$ fetal bovine serum, $50 \mathrm{U} / \mathrm{ml}$ endothelial cell growth factor (Sigma-Aldrich, St. Louis, MO, USA), and 1\% insulintransferrin-selenium (A\&J, Inc., Guangzhou, China).

There were a total of 5 experimental groups in the present study: (group 1) control group (no transfection); (group 2) VEGF transfection (VEGF upregulation); (group 3) VEGF RNA interference (RNAi) (VEGF inhibition); and (group 4) Avastin treatment $(10 \mu \mathrm{g} / \mathrm{ml}$; Genentech, Inc., South San Francisco, CA, USA).

Construction and packaging of rAAV2-VEGF. The coding sequence of the VEGF gene was obtained from the Human cDNA Bank/Domain and synthesized using Golden Taq (Tiangen Biotech, Beijing, China). After plasmid DNA digestion and ligation with the VEGF insert, pSNAV-VEGF plasmids were formed. The transfection of BHK-21 cells with pSNAV-VEGF was performed in a 6 -well plate using a Lipofectamine 2000 kit (Invitrogen, Inc., Carlsbad, CA, USA). These cells are hereafter referred to as BHK/VEGF cells. Next, BHK/VEGF cells were cultured in a flask (110x480 mm; Wheaton, Inc., Millville, NJ, USA), until the cell number reached $8 \times 10^{8}$, and were then infected with HSV1-rc/AUL 2 (Moore=0.1; Benyuan Zhengyang, Inc., Beijing, China) to induce recombinant adeno-associated virus type 2 (rAAV2)-VEGF. After $48 \mathrm{~h}$, cells were detached and divided into Fernbach culture flasks $(250 \mathrm{ml})$ for further purification. Purified rAAV2-VEGF was identified by reverse-transcription polymerase chain reaction (RT-PCR). The titer of rAAV2-VEGF (virus genome/ml) was assessed using hybridization in situ with a digoxin-labeled cucumber mosaic virus probe to obtain a final titer of $1 \times 10^{12}$.

RNAi. A vector containing a small inhibitory RNA for VEGF was constructed by cloning the double-stranded sequence:
Table I. Primers used in real-time PCR analysis.

\begin{tabular}{llc}
\hline Gene & \multicolumn{1}{c}{ Primer $\left(5^{\prime}-3^{\prime}\right)$} & $\begin{array}{c}\text { Annealing } \\
\text { temp }\left({ }^{\circ} \mathrm{C}\right)\end{array}$ \\
\hline VEGF & $\begin{array}{l}\text { CCT CCG AAA CCA TGA ACT TT } \\
\text { TTC TTT GGT CTG CAT TCA CAT T }\end{array}$ & 57 \\
& TCT GGC AGT TCC TTA TGA TAG & 58 \\
& AAA TAC AAC TCC CAT CAC CAG & \\
Ang1 & TTC CTT TCC TTT GCT TTC CTC & 59 \\
& CTGCAGAGCGTTTGTGTTGT & \\
GAPDH & CCT GTA CGC CAA CAC AGT GC & 57 \\
& ATA CTC CTG CTT GCT GAT CC & \\
\hline
\end{tabular}

VEGF, vascular endothelial growth factor; bFGF, basic fibroblast growth factor; Ang1, angiopoietin 1.

5'-gatccAATTTGATATTCATTGATCCGTTCAAGAGACGGATCAATGAATATCAAATTTTTTTTACGCGTg-----3' and 5'-aattcACGCGTAAAAAAAATTTGATATTCATTGATC CGTCTCTTGAACGGATCAATGAATATCAAATTg-----3' (Qiagen, Shanghai, China) into a vector (RNAi-Ready pSIREN-RetroQ ZsGreen pSIREN vector; BD Biosciences, Shanghai, China) to yield the pSIREN-VEGF RNAi vector. The pSIREN-VEGF RNAi or empty pSIREN vectors were transfected into TECs under VEGF stimulation, using the transfection reagent, as previously described (14). Western blotting was performed to examine VEGF expression.

Plasmid transfection. Cultured TECs from 12 patients were divided into 4 groups and transfected with rAAV2-VEGF, pGIPZ-VEGF RNAi, pGIPZ-GFP or rAAV2-GFP, respectively. Cells were plated in 24-well culture plates, grown overnight to $70-80 \%$ confluence, and then washed twice with serum-free basal growth medium. While pGIPZ-GFP or rAAV2-GFP was added to the control group wells (group 1, group 2), $10 \times 10^{9}$ copies of rAAV2-VEGF or PGIPZ-VEGF RNAi were added to each well in the experimental groups. In addition, plasmids containing rAAV2-GFP and pGIPZGFP were separately added to TECs to monitor transfection efficiency.

Real-time PCR. TECs from each experimental group were collected for VEGF, bFGF and ANG1 mRNA expression analysis at 24 and $48 \mathrm{~h}$ by real-time PCR. In brief, total RNA was isolated and reverse transcribed to cDNA using a SuperScript II RT kit (Invitrogen, Inc., Shanghai, China). Real-time PCR was performed using the HotStar Taq Polymerase kit (Qiagen, Inc., Shanghai, China) with SYBR-Green Technology [HSJ200L; Applied Biosystems, Inc., (ABI; Carlsbad, CA, USA)]. Primers for VEGF, bFGF, ANG1 and GAPDH are presented in Table I. PCR reaction was performed in a final reaction volume of $20 \mu 1$ under the following conditions: a preheating cycle at $95^{\circ} \mathrm{C}$ for $5 \mathrm{~min}$, then 33 cycles at $95^{\circ} \mathrm{C}$ for $20 \mathrm{sec}, 56^{\circ} \mathrm{C}$ for $30 \mathrm{sec}$ and $72^{\circ} \mathrm{C}$ for $30 \mathrm{sec}$. Melting curve analysis was performed by monitoring FAM/SYBR fluorescence. 
Western blotting. Total proteins were extracted from transfected TECs and quantitated using the bicinchoninic acid method: $50 \mu \mathrm{g}$ of total protein was mixed with loading buffer, denatured for $5 \mathrm{~min}$ at $60^{\circ} \mathrm{C}$, cooled, centrifuged for $5 \mathrm{~min}$, and separated by sodium dodecyl sulfate polyacrylamide gel electrophoresis and transferred to a polyvinylidene difluoride (PVDF) membrane. Mouse monoclonal antibodies for VEGF $(1,500 x$ dilution; BD Biosciences, San Jose, CA, USA), bFGF, ANG1 (Santa Cruz Biotechnology Inc., Santa Cruz, CA, USA) and GAPDH (Gene Co., Hong Kong, China) were used for probing the proteins. Next, secondary antibody (1,000x dilution) was applied and the signal was revealed by chemiluminescence. The same PVDF membrane was reused to detect $\beta$-actin, which was used as an internal control. Bands observed on the thin films were analyzed by automatic image analysis, and the integrated optical density (OPTDI) of each protein band was normalized to the OPTDI value of the corresponding $\beta$-actin band from the same sample.

Statistical analysis. SAS v.6.12 software (SAS Institute Inc., Cary, NC, USA) was utilized to analyze the expression of VEGF, bFGF, ANG1 and GAPDH in each experimental group, using one-way ANOVA, t-test (unpaired, two-tailed), and Dunnett's multiple comparison test, where appropriate. Results with P-values of $<0.05$ were considered statistically significant.

\section{Results}

VEGF mRNA expression in TECs. Two days after transfection of the TECs, the transfection rate of rAAV2-VEGF was $91 \%$ and that of pGIPZ-VEGF RNAi was $88 \%$ (transfection rates of rAAV2-VEGF and the rAAV2-GFP plasmids were assumed to be equivalent). The results of the real-time PCR mRNA expression analysis are presented in Fig. 1. Significant differences in mRNA expression of VEGF/GAPDH were observed among the 4 transfection groups $(\mathrm{P}<0.0001)$. The highest mRNA expression was detected in the TECs transfected with rAAV2-VEGF. The average VEGF expression level in he rAAV2-VEGF-transfected TECs was 0.58, and the average VEGF expression level in control TECs was 0.20, and this difference in expression was statistically significant $(\mathrm{P}<0.0001)$ (Fig. 1A). The lowest VEGF mRNA expression was detected in the TECs transfected with pGIPZ-VEGF RNAi. The average VEGF expression in the pGIPZ-VEGF RNAi-transfected TECs was 0.10 , and that of the control TECs was 0.23 . This difference in expression was statistically significant $(\mathrm{P}<0.0001)$ (Fig. 1B). Contrary to these findings, no significant difference in VEGF mRNA expression was found between the rAAV2-GFP- and pGIPZ-GFP-transfected TECs $(\mathrm{P}=0.1148)$ (Fig. 1C).

Changes in bFGF and ANG1 expression in response to VEGF level in the TECs. VEGF, bFGF and Ang1 mRNA expression levels were examined 24 and $48 \mathrm{~h}$ after transfection by realtime PCR analysis. The results of these analyses, presented in Fig. 2, respectively, showed that bFGF and Ang1 mRNA expression was decreased when VEGF mRNA expression increased and vice versa $(\mathrm{P}<0.0001)$.
A

B
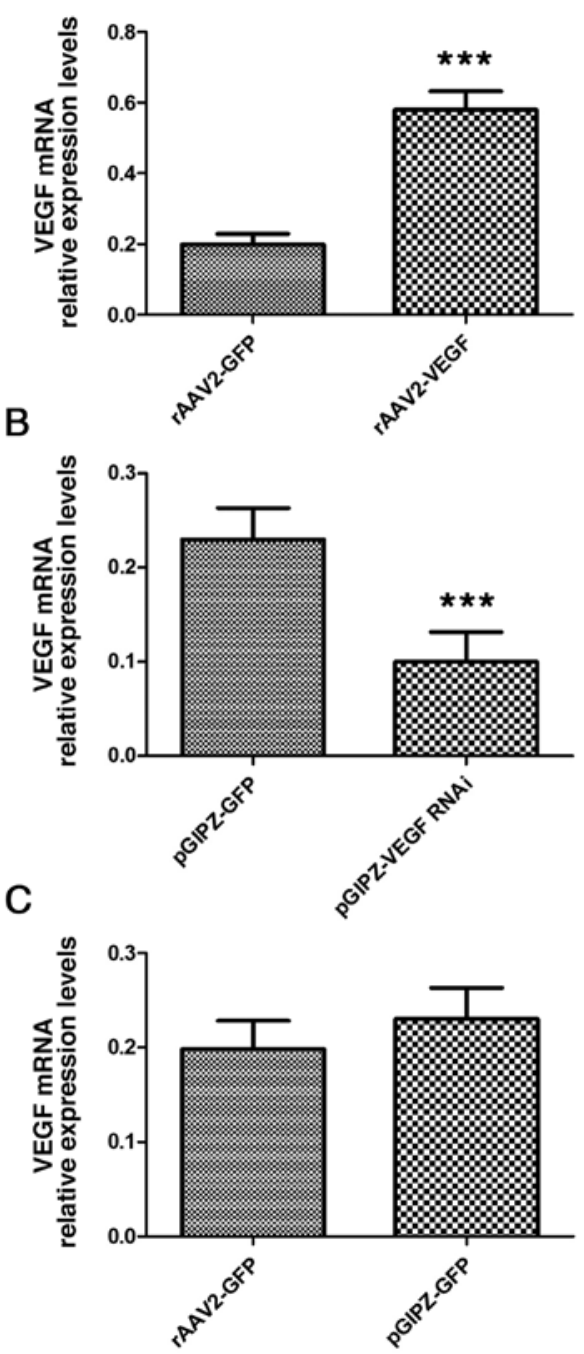

Figure 1. Real-time PCR analysis of VEGF mRNA expression in tumor endothelial cells (TECs) transfected with rAAV2-GFP, rAAV2-VEGF, pGIPZ-GFP or pGIPZ-VEGF RNAi, respectively (mean $\pm S D, n=6$ ). An equal amount of mRNA was used from each group for quantitative RT-PCR and normalized to GAPDH. (A) Significant difference in mRNA expression was found between the rAAV2-GFP and the rAAV2-VEGF groups $\left({ }^{* * *} \mathrm{P}<0.0001\right)$. (B) A significant difference in mRNA expression was found between the pGIPZ-GFP and the pGIPZ-VEGF RNAi groups $\left({ }^{* * *} \mathrm{P}<0.0001\right)$. (C) No significant difference in mRNA expression was found between the rAAV2-GFP and pGIPZ-GFP groups $(\mathrm{P}=0.1148)$.

The average VEGF mRNA expression after 24 or $48 \mathrm{~h}$ was 0.28 and 0.24 , respectively, in the control groups, 0.71 and 0.73 , respectively, in the rAAV2-VEGF groups, and 0.57 and 0.51 , respectively, in the Avastin-treated groups. When the experimental groups were compared with their respective control groups after 24 or $48 \mathrm{~h}$, using Dunnett's multiple comparison test, the observed differences in mRNA expression were found to be statistically significant (Figs. $2 \mathrm{~A}$ and $3 \mathrm{~A}$, respectively).

The average bFGF mRNA expression in the control groups after 24 and $48 \mathrm{~h}$ was 0.35 and 0.35 , respectively; in the rAAV2-VEGF groups after 24 and $48 \mathrm{~h}$, this level was 0.24 and 0.26 , respectively; in the pGIPZ-VEGF RNAi groups after 24 and $48 \mathrm{~h}$, the average bFGF mRNA expression was 1.07 and 1.10, respectively; and in the Avastin groups after 24 and 
A
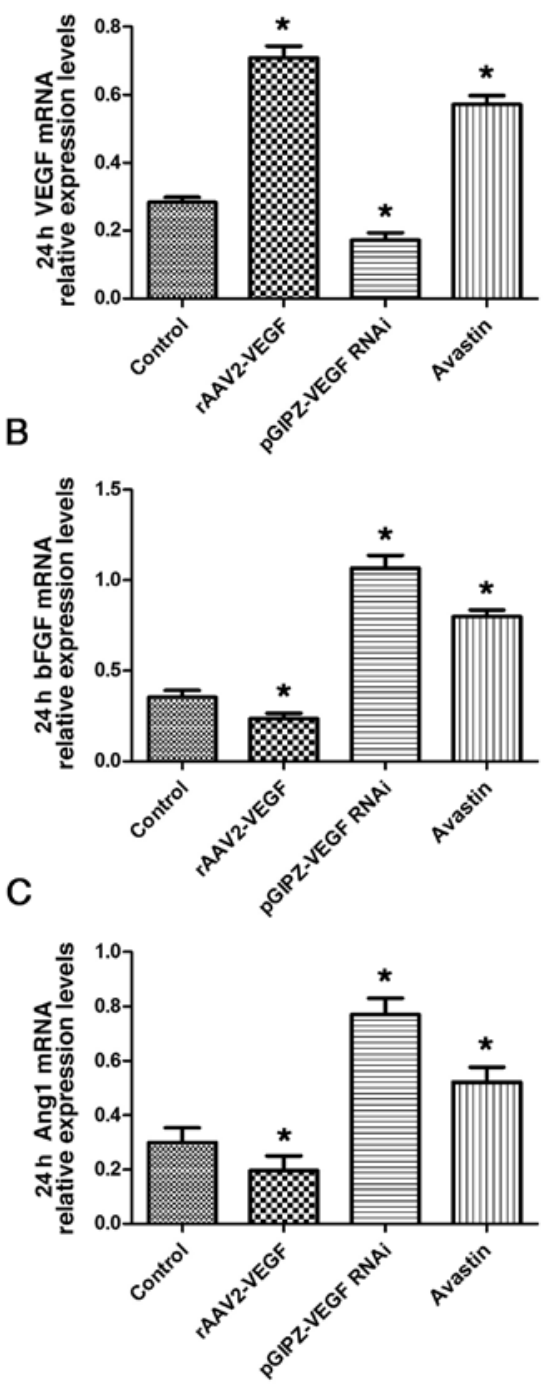

Figure 2. Real-time PCR analysis of VEGF, bFGF and Ang1 mRNA expression in untreated tumor endothelial cells (TECs) (group 1), rAAV2-VEGF-treated TECs (group 2), pGIPZ-VEGF RNAi-treated TECs (group 3) and Avastin-treated cells (group 4) after $24 \mathrm{~h}$ (mean \pm SD, $\mathrm{n}=6$ ). An equal amount of mRNA was used from each group for quantitative RT-PCR and normalized to GAPDH. (A) Significant differences in VEGF mRNA expression were found between the experimental and control groups $\left({ }^{*} \mathrm{P}<0.05\right)$. (B) Significant differences in bFGF mRNA expression were found between the experimental and control groups $(" \mathrm{P}<0.05)$. (C) Significant differences in Ang1 mRNA expression were found between the experimental and control groups $\left({ }^{*} \mathrm{P}<0.05\right)$.

$48 \mathrm{~h}$, it was 0.80 and 0.78 , respectively. When the experimental groups were compared with their respective control groups after 24 or 48 h by Dunnett's multiple comparison test, the observed differences in mRNA expression were found to be statistically significant (Figs. 2B and 3B, respectively).

Average Ang1 mRNA expression in the control groups after 24 and $48 \mathrm{~h}$ was 0.30 and 0.32 , respectively; in rAAV2-VEGF groups after 24 and $48 \mathrm{~h}$, it was 0.20 and 0.26 , respectively; in pGIPZ-VEGF RNA groups after 24 and $48 \mathrm{~h}$, it was 0.77 and 0.82 , respectively; and in Avastin groups after 24 and $48 \mathrm{~h}$, it was 0.52 and 0.54 , respectively. When experimental groups were compared with their respective control groups after 24 or $48 \mathrm{~h}$ by Dunnett's multiple comparison test, the observed
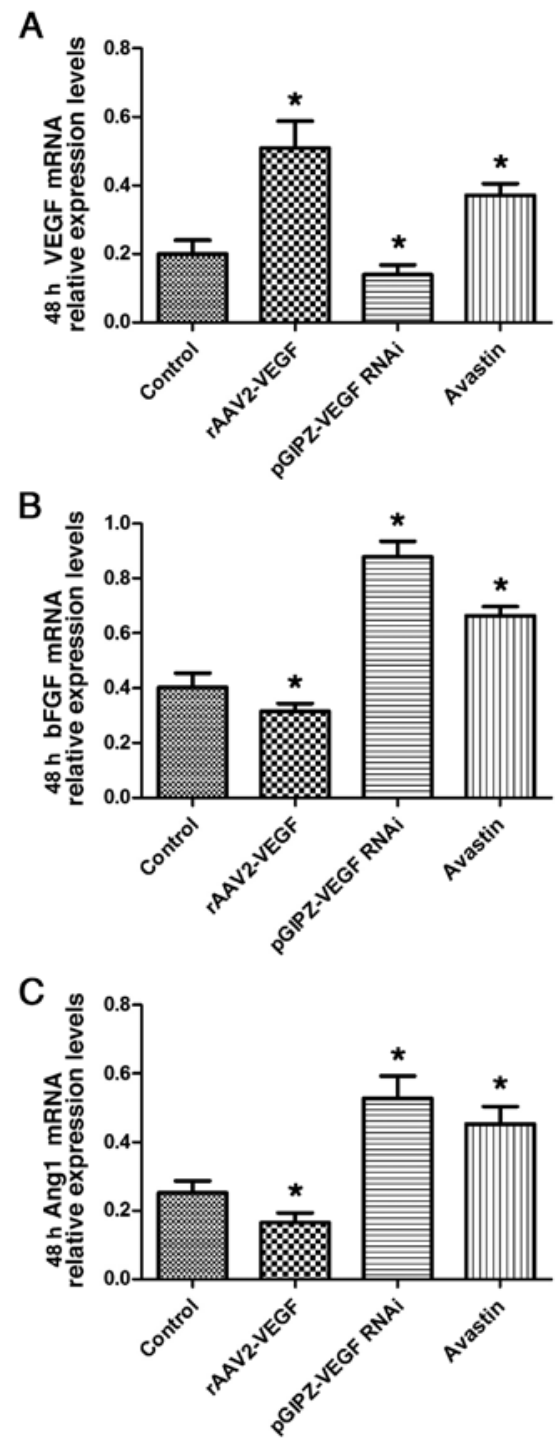

Figure 3. Real-time PCR analysis of VEGF, bFGF and Ang1 mRNA expression in untreated tumor endothelial cells (TECs) (group 1), rAAV2-VEGF-treated TECs (group 2), pGIPZ-VEGF RNAi-treated TECs (group 3) and Avastin-treated cells (group 4) after $48 \mathrm{~h}$ (mean \pm SD, $\mathrm{n}=6$ ). An equal amount of mRNA was used from each group for quantitative RT-PCR and normalized to GAPDH. (A) Significant differences in VEGF mRNA expression were found between experimental and control groups $\left({ }^{*} \mathrm{P}<0.05\right)$. (B) Significant differences in bFGF mRNA expression were found between the experimental and control groups $\left({ }^{*} \mathrm{P}<0.05\right)$. (C) Significant differences in Ang1 mRNA expression were found between the experimental and control groups $\left({ }^{*} \mathrm{P}<0.05\right)$.

differences in mRNA expression were found to be statistically significant (Figs. 2C and 3C, respectively).

Protein expression after 24 and $48 \mathrm{~h}$ was examined by western blotting (Figs. 4A and 5A, respectively). The expression of bFGF and ANG1 proteins was decreased when VEGF protein expression was increased and vice versa $(\mathrm{P}<0.0001)$.

The average VEGF protein expression in the control groups after 24 or $48 \mathrm{~h}$ was 0.20 and 0.21 , respectively. The average VEGF protein expression in the rAAV2-VEGF groups was 0.51 and 0.62 , respectively, and in the pGIPZ-VEGF RNAi groups it was 0.14 and 0.16 , respectively. In the Avastin groups, the level was 0.37 and 0.41 , respectively. When the experimental groups were compared with their respective control groups 
A

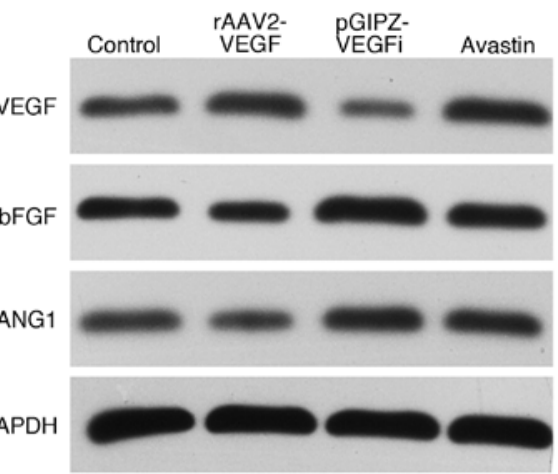

C

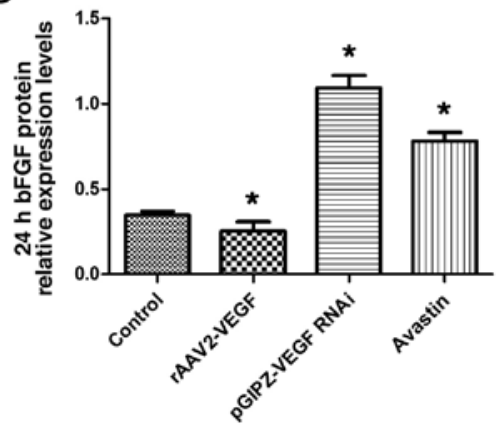

B

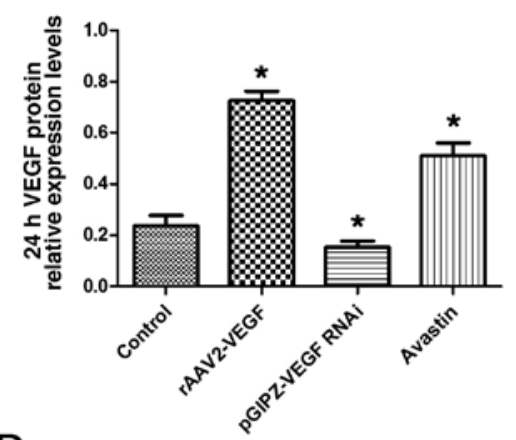

D

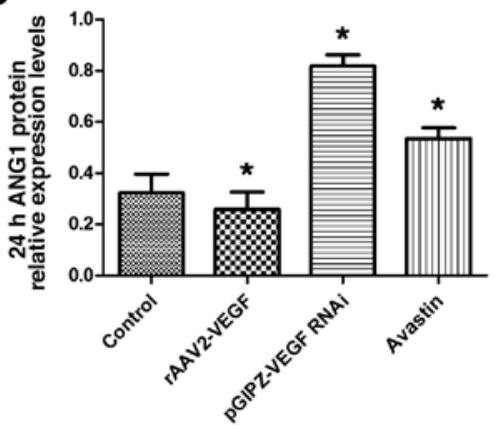

Figure 4. Western blot analysis of VEGF, bFGF and ANG1 protein expression in untreated tumor endothelial cells (TECs) (group 1), rAAV2-VEGF-treated TECs (group 2), pGIPZ-VEGF RNAi-treated TECs (group 3), and Avastin-treated cells (group 4) after $24 \mathrm{~h}$ (mean \pm SD, n=6), where density values were compared to GAPDH density. (A) Western blotting representing VEGF, bFGF and ANG1 protein at $24 \mathrm{~h}$. (B) Significant differences in VEGF protein expression were found between experimental and control groups $\left({ }^{*} \mathrm{P}<0.05\right)$. (C) Significant differences in bFGF protein expression were found between experimental groups ( $(\mathrm{P}<0.05)$. (D) Significant differences in ANG1 protein expression were found between (experimental and control groups (" $\mathrm{P}<0.05)$.

A

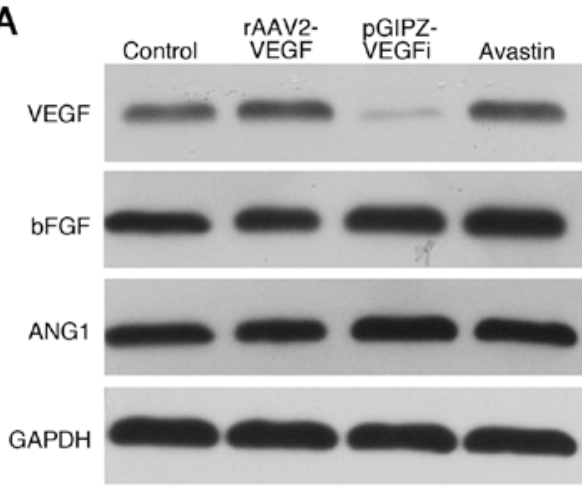

C

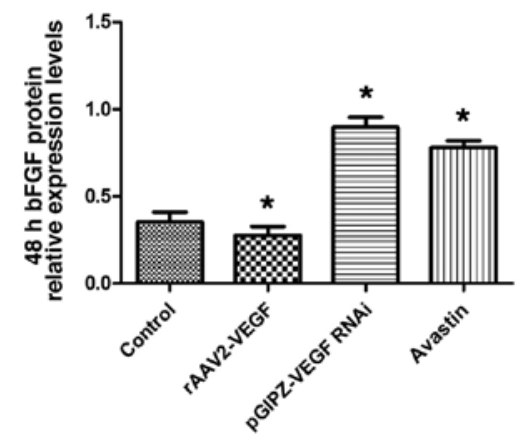

B

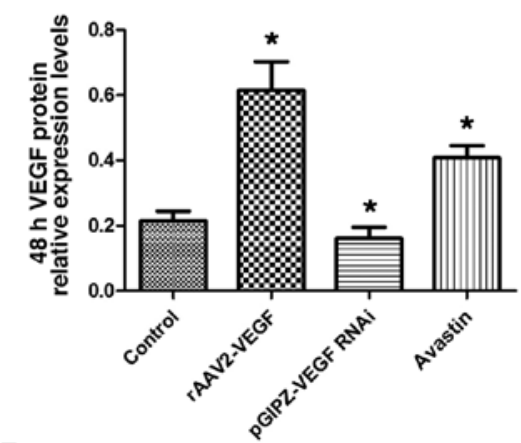

D

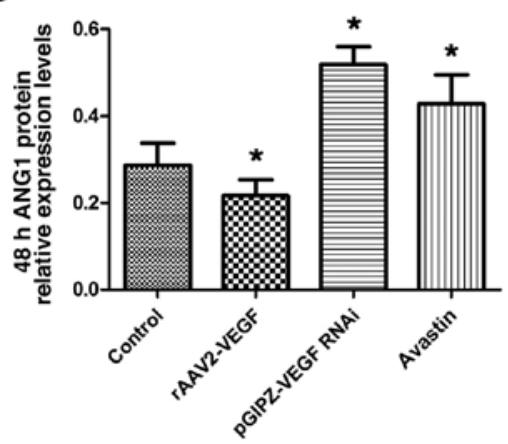

Figure 5. Western blot analysis of VEGF, bFGF and ANG1 protein expression in untreated tumor endothelial cells (TECs) (group 1), rAAV2-VEGF-treated TECs (group 2), pGIPZ-VEGF RNAi-treated TECs (group 3) and Avastin-treated cells (group 4) after $48 \mathrm{~h}$ (mean $\pm \mathrm{SD}$, $\mathrm{n}=6$ ), where density values were compared to GAPDH density. (A) Western blotting representing VEGF, bFGF, and ANG1 protein expression at 48 h. (B) Significant differences in VEGF protein expression were found between experimental and control groups ( $\mathrm{P}<0.05)$. (C) Significant differences in bFGF protein expression were found between experimental and control groups ( $\mathrm{P}<0.05)$. (D) Significant differences in ANG1 protein expression were found between experimental and control groups $\left({ }^{*} \mathrm{P}<0.05\right)$. 
after 24 or 48 h by Dunnett's multiple comparison test, the observed differences in protein expression were found to be significant (Figs. 4B and 5B, respectively).

The average bFGF protein expression in the control groups at 24 and $48 \mathrm{~h}$ was 0.40 and 0.35 , respectively; in the rAAV2-VEGF groups after 24 and $48 \mathrm{~h}$ it was 0.32 and 0.28 , respectively; in the pGIPZ-VEGF RNAi groups after 24 and $48 \mathrm{~h}$ it was 0.88 and 0.90 , respectively; and in the Avastintreated 24 and $48 \mathrm{~h}$ groups 0.67 and 0.78 , respectively. When the experimental groups were compared with their respective control groups after 24 or $48 \mathrm{~h}$ by Dunnett's multiple comparison test, the observed differences in protein expression were found to be statistically significant (Figs. 4C and 5C, respectively).

The average ANG1 protein expression in the control groups at 24 and $48 \mathrm{~h}$ was 0.25 and 0.29 , respectively; in rAAV2-VEGF groups after 24 and $48 \mathrm{~h}$, it was 0.17 and 0.22 , respectively; in the pGIPZ-VEGF RNAi groups after 24 and $48 \mathrm{~h}$, it was 0.53 and 0.52 , respectively; and in the Avastin groups after 24 and $48 \mathrm{~h}$, it was 0.45 and 0.43 , respectively. When the experimental groups were compared with their respective control groups after 24 or $48 \mathrm{~h}$ by Dunnett's multiple comparison test, the observed differences in protein expression were found to be statistically significant (Figs. 4D and 5D, respectively).

\section{Discussion}

Tumor angiogenesis is a key process in tumor development and progression. Tumor angiogenesis is important in the early stages of tumorigenesis when the tumor grows beyond the size where simple diffusion is sufficient for oxygen and nutrient delivery and new vessels are needed to perform this role $(17,18)$. In later stages, angiogenesis is essential for tumor spread and metastasis; these characteristics make it an attractive target for antitumor therapy (18). Angiogenesis is a complex process with many different molecules involved in the maintenance of the delicate balance between pro-angiogenic and antiangiogenic factors, with VEGF being one of the key master regulators of this dynamic homeostasis $(19,20)$. Indeed, the first antitumor therapy targeting angiogenesis was developed more than a decade ago using monoclonal antibodies, namely bevacizumab directed against VEGF (21).

Currently, bevacizumab is commonly used along with conventional chemotherapy in the first-line treatment of metastatic colorectal cancer; however, it is used with only modest improvements in patient progression and overall survival when compared to chemotherapy alone. Furthermore, some patients do not benefit at all from this therapy (5,6,22-24).

The human organism is a complex system and most of its features result from the organic organization of many regulatory and signaling networks of molecules. Self-regulating mechanisms are crucial to maintain its structural stability and normal function. Although the role of many different signaling molecules and growth factors in angiogenesis has been studied previously, our understanding of how a particular factor functions still does not entirely clarify the new features emerging only as a result of complex interactions of a higher order between molecules constituting different regulatory networks. Recently, approaches of systems biology have been applied in the research of self-regulating mechanisms, and the results are exciting $(20,25)$. The main regulatory mechanism for the maintenance of organism stability is nerve-body fluid-immunoregulation (12). The same mechanism can be observed in other physiological processes, including angiogenesis. Indeed, when key angiogenic factors fail in their physiological function or are inhibited, other factors are able to compensate for their function (22).

Therefore, in the present study we examined VEGF as the key regulatory switch in angiogenesis and clarified the compensatory function of other vessel growth factors in endothelial cells derived from colon cancer. It has been shown that bFGF and ANG1 act as positive effectors of angiogenesis $(4,23,24,26)$, with the function of promoting vascular proliferation.

ANG1 is responsible for the maintenance of endothelial cell stability: it inhibits VEGF-mediated angiogenesis, and it has been shown in previous studies that overexpression of ANG1 is correlated with high tumor microvessel density in colorectal cancer (27).

bFGF has been reported to promote cell growth, migration and angiogenesis (15). Its aberrant expression has been described in tumor angiogenesis and it has been pinpointed as a potential cause of resistance to anti-VEGF therapy (28).

In the present study, when VEGF expression was upregulated in TECs, the expression levels of bFGF and ANG1 were downregulated. However, when VEGF expression was inhibited, the function of VEGF was compensated by upregulation of bFGF and ANG1. Therefore, it seems that VEGF, bFGF and ANG1 constitute a self-regulating mechanism in colon cancer angiogenesis.

After TECs were treated with bevacizumab, VEGF expression was significantly higher than that noted in the control group. This may be explained by bevacizumab inhibiting the physical function of VEGF, which may trigger the compensatory VEGF self-regulating mechanisms. It seems that other vascular growth factors compensate for the function of VEGF to some degree as well and that therefore the angiogenesis cannot be completely inhibited by bevacizumab.

Indeed, it has been shown that in the normal balanced state of an organism, autocrine VEGF is required for the homeostasis of blood vessels (29-31). When VEGF expression is not adequate, self-regulating vascular growth/anagogic factor mechanisms are able to compensate for the function of VEGF, at least in part, and maintain the stability of blood vessels. In the present study, we demonstrated that when endothelial cells derived from colon cancer were treated with bevacizumab, the resulting VEGF inhibition may also increase the expression of other growth factors to compensate for the loss of VEGF, due to the existence of a self-regulating mechanism that weakens its therapeutic efficiency.

In conclusion, our data provide evidence on the compensatory self-regulating mechanisms in bevacizumab-treated endothelial cells derived from colon cancer. We believe that it is essential to bring new ideas to the development of antiangiogenic targeted therapy for colon cancer and, potentially, to target more than just VEGF to inhibit collateral networks that maintain tumor angiogenesis.

\section{Acknowledgements}

The present study was approved by the Ethics Committee and Institutional Review Board of the Central Hospital of 
Ezhou. The present study was supported by the National Nature Science Foundation (China) (grant nos. 30872823 and 30672278).

\section{References}

1. Muzny DM, Bainbridge MN, Chang K, Dinh HH, Drummond JA, Fowler G, Kovar CL, Lewis LR, Morgan MB, Newsham IF, et al; Cancer Genome Atlas Network: Comprehensive molecular characterization of human colon and rectal cancer. Nature 487: 330-337, 2012

2. Yadav L, Puri N, Rastogi V, Satpute P and Sharma V: Tumour Angiogenesis and Angiogenic Inhibitors: A Review. J Clin Diagn Res 9: XE01-XE05, 2015.

3. Papadimitriou K, Rolfo C, Dewaele E, Van De Wiel M, Van den Brande J, Altintas S, Huizing M, Specenier P and Peeters M: Incorporating anti-VEGF pathway therapy as a continuum of care in metastatic colorectal cancer. Curr Treat Options Oncol 16: 18, 2015.

4. Hurwitz H, Fehrenbacher L, Novotny W, Cartwright T, Hainsworth J, Heim W, Berlin J, Baron A, Griffing S, Holmgren E, et al: Bevacizumab plus irinotecan, fluorouracil, and leucovorin for metastatic colorectal cancer. N Engl J Med 350: 2335-2342, 2004.

5. Saltz LB, Clarke S, Díaz-Rubio E, Scheithauer W, Figer A, Wong R, Koski S, Lichinitser M, Yang TS, Rivera F, et al: Bevacizumab in combination with oxaliplatin-based chemotherapy as first-line therapy in metastatic colorectal cancer: A randomized phase III study. J Clin Oncol 26: 2013-2019, 2008.

6. Tebbutt NC, Wilson K, Gebski VJ, Cummins MM, Zannino D van Hazel GA, Robinson B, Broad A, Ganju V, Ackland SP, et al: Capecitabine, bevacizumab, and mitomycin in first-line treatment of metastatic colorectal cancer: Results of the Australasian Gastrointestinal Trials Group Randomized Phase III MAX Study. J Clin Oncol 28: 3191-3198, 2010.

7. Grothey A and Ellis LM: Targeting angiogenesis driven by vascular endothelial growth factors using antibody-based therapies. Cancer J 14: 170-177, 2008.

8. Shibuya M: Vascular endothelial growth factor-dependent and -independent regulation of angiogenesis. BMB Rep 41: 278-286, 2008

9. Fleck BW and McIntosh N: Pathogenesis of retinopathy of prematurity and possible preventive strategies. Early Hum Dev 84: 83-88, 2008.

10. Heidary G, Vanderveen D and Smith LE: Retinopathy of prematurity: Current concepts in molecular pathogenesis. Semin Ophthalmol 24: 77-81, 2009.

11. Karamysheva AF: Mechanisms of angiogenesis. Biochemistry 73: 751-762, 2008.

12. Kitano H: Cancer as a robust system: Implications for anticancer therapy. Nat Rev Cancer 4: 227-235, 2004.

13. Schrattenholz A and Soskić V: What does systems biology mean for drug development? Curr Med Chem 15: 1520-1528, 2008
14. Li B, Li GG, Xiang Y and Xu LJ: VEGF affects the expression of TGF- $\beta$ in retinal neovascularization: A preliminary study about redundancy of growth factor. Int J Ophthalmol 8: 2391-2393, 2008.

15. Gacche RN and Meshram RJ: Angiogenic factors as potential drug target: Efficacy and limitations of anti-angiogenic therapy. Biochim Biophys Acta 1846: 161-179, 2014.

16. Kerbel RS: Tumor angiogenesis. N Engl J Med 358: 2039-2049, 2008.

17. Giampieri R, Scartozzi M, Del Prete M, Fulli A, Faloppi L, Bianconi M, Maccaroni E and Cascinu S: The 'angiogenetic ladder', step-wise angiogenesis inhibition in metastatic colorectal cancer. Cancer Treat Rev 40: 934-941, 2014.

18. Hanahan D and Weinberg RA: The hallmarks of cancer. Cell 100: 57-70, 2000.

19. Mahadevan R and Lovley DR: The degree of redundancy in metabolic genes is linked to mode of metabolism. Biophys J 94: $1216-1220,2008$

20. Krakauer DC and Plotkin JB: Redundancy, antiredundancy, and the robustness of genomes. Proc Natl Acad Sci USA 99: 1405-1409, 2002

21. Louis EJ: Evolutionary genetics: Making the most of redundancy. Nature 449: 673-674, 2007.

22. Saharinen P, Eklund L, Miettinen J, Wirkkala R, Anisimov A, Winderlich M, Nottebaum A, Vestweber D, Deutsch U, Koh GY, et al: Angiopoietins assemble distinct Tie2 signalling complexes in endothelial cell-cell and cell-matrix contacts. Nat Cell Biol 10: 527-537, 2008.

23. Cao Y and Liu Q: Therapeutic targets of multiple angiogenic factors for the treatment of cancer and metastasis. Adv Cancer Res 97: 203-224, 2007.

24. Wong ET and Brem S: Antiangiogenesis treatment for glioblastoma multiforme: Challenges and opportunities. J Natl Compr Canc Netw 6: 515-522, 2008.

25. Moens S, Goveia J, Stapor PC, Cantelmo AR and Carmeliet P: The multifaceted activity of VEGF in angiogenesis - Implications for therapy responses. Cytokine Growth Factor Rev 25: 473-482, 2014.

26. Tabernero J: The role of VEGF and EGFR inhibition: Implications for combining anti-VEGF and anti-EGFR agents. Mol Cancer Res 5: 203-220, 2007.

27. Chung YC, Hou YC, Chang $\mathrm{CN}$ and Hseu TH: Expression and prognostic significance of angiopoietin in colorectal carcinoma. J Surg Oncol 94: 631-638, 2006.

28. Lieu C, Heymach J, Overman M, Tran H and Kopetz S: Beyond VEGF: Inhibition of the fibroblast growth factor pathway and antiangiogenesis. Clin Cancer Res 17: 6130-6139, 2011.

29. Yancopoulos GD, Davis S, Gale NW, Rudge JS, Wiegand SJ and Holash J: Vascular-specific growth factors and blood vessel formation. Nature 407: 242-248, 2000.

30. Dvorak HF: Discovery of vascular permeability factor (VPF). Exp Cell Res 312: 522-526, 2006.

31. Folkman J: Angiogenesis: An organizing principle for drug discovery? Nat Rev Drug Discov 6: 273-286, 2007. 